\title{
Skeletal muscle loss was associated with the risk of diabetes in non non-alcoholic fatty liver disease Chinese male middle-aged and elderly population, the Shanghai Changfeng Study
}

\section{Lingyan Chen ( $\sim$ chlingyan1984@163.com )}

Zhongshan Hospital Fudan University https://orcid.org/0000-0002-5347-1549

\section{Mingfeng Xia}

Zhongshan Hospital Fudan University

\section{Li Wu}

Zhongshan Hospital Fudan University

\section{Qian Li}

Zhongshan Hospital Fudan University

\section{Yu Hu}

Zhongshan Hospital Fudan University

Hui Ma

Zhongshan Hospital Fudan University

\section{Xin Gao}

Zhongshan Hospital Fudan University

\section{Huandong Lin}

Zhongshan Hospital Fudan University

\section{Research}

Keywords: skeletal muscle mass, diabetes mellitus, NAFLD, liver fat content

Posted Date: March 24th, 2021

DOI: https://doi.org/10.21203/rs.3.rs-351690/v1

License: (c) (i) This work is licensed under a Creative Commons Attribution 4.0 International License. Read Full License 


\section{Abstract \\ Background}

Previous studies have presented skeletal muscle loss was associated with diabetes mellitus (DM) and non-alcoholic fatty liver disease (NAFLD). However, whether the presence of NAFLD could influence the association between skeletal muscle mass and DM was still unknown. The aim of the present study was to investigate the relationship of skeletal muscle mass with diabetes in Chinese middle-aged and older community population, and whether the association could be effected by NAFLD.

\section{Methods}

A cross-sectional study of 5,626 residents aged 45 and above in Changfeng community in Shanghai were conducted. Skeletal muscle mass (SMM) was detected by dual-energy $X$ ray absorption (DXA) and calculated as ASM\% [appendicular skeletal muscle mass (ASM) (kg)/body weight*100\%]. Liver fat content (LFC) was measured using a quantitative ultrasound method. Multivariate logistic regression analyses were conducted to investigate the association between ASM\% quartiles with DM.

\section{Results}

With ASM\% decreasing, fasting blood glucose (FBG), 2-hour postprandial blood glucose (2hBG) and Homeostasis model assessment for insulin resistance (HOMA-IR) as well as LFC increased in both genders, and the prevalence of diabetes and NAFLD also increased. Spearman analysis showed ASM\% was negatively correlated with FBG, $2 \mathrm{hBG}$ and LFC. Stepwise logistic regression analysis showed after multiple adjustments, ASM\% quartile was negatively associated with the risk of diabetes only in male, but not in female. Subgroup analysis found the ASM\% quartiles remained negatively correlated with the risk of diabetes in male non-NAFLD population, but not in NAFLD population. When stratified by LFC quartiles, the results was similar. After multivariate adjustments, low ASM\% was negatively correlated with the risk of diabetes only in the first and second LFC quartile in male, and the association remained insignificant in each LFC quartile in female.

\section{Conclusions}

Skeletal muscle mass was negatively associated with the risk of diabetes in male population but not in female. The presence of NAFLD weakened this association. The results suggested stratified management of diabetes should be considered according to skeletal muscle mass and the presence of NAFLD.

\section{Background}


With the age increasing, the progressive decreasing in skeletal muscle mass and strength, which was called sarcopenia has significantly increased ${ }^{[1]}$. Skeletal muscle loss reduced the mobility of the elderly, increased the risk of fractures and falls, and meanwhile was closely related to metabolic disorders, tumors and other chronic diseases ${ }^{[2-4]}$. With the aggravation of the aging society, sarcopenia has become an important worldwide public health problem.

As the largest non-fat component of the human body, skeletal muscle accounts for about $40 \%$ of body weight and is responsible for most of the postprandial glucose disposition. As an important insulin target organ for glucose uptake and utilization, skeletal muscle loss with insulin resistance would lead to systemic metabolic disorder, which was closely related to diabetes ${ }^{[5-7]}$. Compared with non-diabetics, diabetic patients had lower muscle mass and higher prevalence of sarcopenia ${ }^{[8]}$. Conversely, reduced skeletal muscle mass may also increase the risk of diabetes ${ }^{[9]}$. The results in previous few studies about the relationship between skeletal muscle mass and the risk of diabetes were not consistent ${ }^{[9,10]}$, which may due to different ethnic, gender, age and other population characteristics.

NAFLD is a chronic liver disease caused by abnormal accumulation of fat in the liver. Previous studies have shown type 2 diabetes often coexists with the occurrence and progression of NAFLD. In China, about $28-70 \%$ of type 2 diabetes patients have NALFD, and meanwhile $22.5 \%$ of NAFLD patients suffered from type 2 diabetes ${ }^{[11,12]}$. The risk of diabetes also increased with the progression of liver disease $^{[13]}$. All the above suggested type 2 diabetes was closely related to NAFLD. As muscle and liver are both important target organs of insulin action and effect significantly on maintaining glucose homeostasis, which indicated skeletal muscle had an important role on the development of both diabetes and NAFLD. Indeed, a few previous studies have shown that age-related skeletal muscle mass reduction was associated with NAFLD, NASH (non-alcoholic steatohepatitis), and related liver fibrosis ${ }^{[14-16]}$.

However, so far no research was conducted to explore the relationship between skeletal muscle mass and the risk of diabetes in Chinese community population. Furthermore, whether NAFLD, which was closely related to both skeletal muscle mass and diabetes, would effect significantly on this relationship remains unknown. Therefore, it is necessary to carry out a large-scale community population study to investigate the association of skeletal muscle mass, DM and NAFLD.

In the present study, we recruited participants aged 45 and above of Changfeng Community in Shanghai to investigate the relationship between skeletal muscle mass and diabetes, as well as the role of NAFLD involved in the relationship, in order to provide new evidences for the prevention and treatment of NAFLD and diabetes.

\section{Methods}

\section{Participants}


we enrolled a total of 5626 residents aged 45 and above of Changfeng community in Shanghai from May 2010 to December $2012^{[17,18]}$. Participants according with the following criteria were excluded: 1 ) lacking biochemical and liver fat content data; 2) lacking DXA data; 3) viral hepatitis and excessive alcohol consumption. Finally, 3969 subjects were included in the analysis (1370 men and 2599 women).

All participants were informed of the research details and signed the informed consent. The study was approved by the Ethics Committee of Zhongshan Hospital of Fudan University.

\section{Data collection}

Trained researchers interviewed all participants and obtained their medical history by a standard questionnaire. Then, standing height and body weight were measured without shoes and outer clothing. Body mass index (BMI) was calculated as weight divided by height squared $\left(\mathrm{kg} / \mathrm{m}^{2}\right)$. Resting blood pressure (BP) including systolic blood pressure (SBP) and diastolic blood pressure (DBP) were measured three times with an electronic blood pressure monitor (OMRON Model HEM-752 FUZZY, Omron Co., Dalian, China), and the average was calculated. All the above has been previously described elsewhere ${ }^{[17,18]}$.

Blood samples were collected after a fasting period of at least 10 hours overnight. Biochemical indexes including FBG, total cholesterol (TC), triglycerides (TG), high-density lipoprotein-cholesterol (HDL-C) were measured with an automated bio-analyzer (HITACHI 7600, Tokyo, Japan). Low-density lipoprotein cholesterol (LDL-C) was calculated using the Friedewald equation. $2 \mathrm{hBG}$ was tested following a $75 \mathrm{~g}$ oral glucose tolerance for non-diabetics or a $100 \mathrm{~g}$ steamed bread meal for diagnosed diabetes.

Electrochemiluminescence immunoassay was used to measure the serum insulin concentrations. HOMAIR was calculated by multiplying the FBG $(\mathrm{mmol} / \mathrm{L})$ times fasting insulin $(\mathrm{mU} / \mathrm{L})$ and dividing by 22.5 .

Hepatic ultrasonography scanning was performed by an experienced technician who was blinded to the participants' details using a GE Logiq P5 scanner (GE Healthcare, Milwaukee,USA) with a 4-MHz probe. The liver ultrasound images were analysed with imaging software from the National Institutes of Health (Image J 1.410, National Institutes of Health, Bethesda, MD) and standardised using a tissue-mimicking phantom (Model 057; Computerized Imaging Reference Systems, Norfolk, VA). We measured LFC according to the method described in detail elsewhere ${ }^{[19]}$.

Body composition including lean mass and fat mass (FM) were measured using dual-energy $\mathrm{X}$-ray absorptiometry (Lunar iDXA, GE Healthcare). All measurements were carried out by a single, trained technician at a single clinical center. Manual DXA analysis software was used to analyze all of the DXA scans. The percentage of fat mass (FM\%) was calculated as FM divided by total body mass. SMM was calculated as following: weight adjusted by appendicular skeletal muscle mass (ASM\% = appendicular skeletal muscle mass $(\mathrm{kg}) /$ body weight $(\mathrm{kg}) \times 100 \%)^{[9,20]}$.

\section{Definitions}


Obesity was defined as $\mathrm{BMI} \geqq 28 \mathrm{~kg} / \mathrm{m}^{2}$ by Chinese criterion ${ }^{[21]}$. Diabetes mellitus (DM) was defined as $\mathrm{FBG} \geqq 7.0 \mathrm{mmol} / \mathrm{I}$ or $2 \mathrm{hBG} \geqq 11.1 \mathrm{mmoo} / \mathrm{l}$ based on OGTT by WHO 1999 criteria ${ }^{[22]}$ or a previous diagnosis or self reported current hypoglycemic treatment. NAFLD was diagnosed when LFC by ultrasonography exceeded the cut-off value of $9.15 \%$, excluding excessive alcoholic intake and virus hepatitis ${ }^{[19]}$.

\section{Statistical analysis}

All statistical analysis were performed using SPSS software version 19.0 (SPSS, Chicago, IL, USA). The continuous data are presented as the means \pm SD except for skewed variables, which were presented as the median with the inter-quartile range (25-75\%) given in parentheses. All subjects were divided into four groups according to gender-specific quartiles of ASM\% as follows: male区Q1: $\geq 32.0 \%, \mathrm{Q} 2: \geq 30.5 \% \sim \rrbracket$

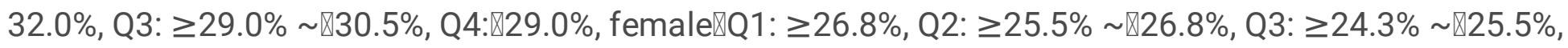
$\mathrm{Q} 4: 囚 24.3 \%$. Analysis of variance (ANOVA) or the Kruskal-Wallis test was used for inter-group comparisons of continuous data, whereas the Chi-squared test was used for comparisons of categorical variables. Spearman analysis was performed to assess the relationships between ASM\% and blood glucose as well as other clinical parameters. Multivariate logistic regression analyses were conducted to investigate the association between ASM\% quartiles with DM after successively adjusting for age, cigarette smoking, diabetes family history, fat mass, interaction between fat mass and ASM\% quartiles, the presence of obesity, blood pressure, serum triglyceride, HDL-C, and HOMA-IR. The interaction between ASM\% and fat mass was included in the multiple regression models because there were significant correlations between ASM\%, fat mass and blood glucose. To further investigate whether NAFLD effected the relationship between SMM and DM, subgroup analysis were performed according to the presence of NAFLD and LFC quartiles. $P$ value $<0.05$ was considered to be statistically significant.

\section{Results}

\section{Characteristics of subjects}

Of the total 3969 subjects, the mean age was 63.3 years, and the mean BMI was $24.1 \mathrm{~kg} / \mathrm{m}^{2}$. Details of the subject characteristics are shown in Table 1. All subjects with lower ASM\% were older and had higher body weigh and blood pressure, with higher BMI, FM, FM\%, SBP and DBP. The lipid disorders were aggravated in subjects with lower ASM\%, presenting higher TC, TC and LDL-C, and lower HDL-C. The most noteworthy was that FBG, 2hBG, HOMA-IR and LFC increased gradually as well as the prevalence of DM and NAFLD, with ASM\% decreasing in both male and female participants (all $P<0.001$ ). 
Table 1

Characteristics of male participants according to the ASM\% quartiles

\section{Quartiles of ASM\%}

$P$
value

\begin{tabular}{|c|c|c|c|c|c|}
\hline Male & Q1 $n=343$ & Q2 n = 343 & Q3 n = 343 & $\begin{array}{l}\text { Q4 } \\
n=342\end{array}$ & \\
\hline Age( year) & $61.6 \pm 8.8$ & $63.2 \pm 8.9^{*}$ & $63.2 \pm 8.9^{*}$ & $69.4 \pm 10.1^{*}$ & $<0.001$ \\
\hline BMI $\left(\mathrm{kg} / \mathrm{m}^{2}\right)$ & $22.7 \pm 2.9$ & $24.3 \pm 3.4^{\star}$ & $24.3 \pm 3.4^{*}$ & $25.6 \pm 2.9^{*}$ & $<0.001$ \\
\hline WC, $\mathrm{cm}$ & $80.6 \pm 9.1$ & $85.8 \pm 7.7^{\star}$ & $85.8 \pm 7.7^{\star}$ & $91.7 \pm 8.5^{\star}$ & $<0.001$ \\
\hline $\mathrm{FM}, \mathrm{kg}$ & $17.9 \pm 4.6$ & $20.3 \pm 4.3^{*}$ & $20.3 \pm 4.3^{*}$ & $24.5 \pm 4.9^{*}$ & $<0.001$ \\
\hline FM\% & $22.4 \pm 4.2$ & $27.2 \pm 3.2^{*}$ & $27.2 \pm 3.2^{*}$ & $33.1 \pm 3.3^{*}$ & $<0.001$ \\
\hline ASM\% & $32.6 \pm 1.9$ & $30.6 \pm 1.0^{\star}$ & $30.6 \pm 1.0^{\star}$ & $27.2 \pm 1.6^{\star}$ & $<0.001$ \\
\hline SBP, mmHg & $129 \pm 18$ & $134 \pm 17^{*}$ & $134 \pm 17^{\star}$ & $141 \pm 19^{*}$ & $<0.001$ \\
\hline DBP, mmHg & $76 \pm 11$ & $78 \pm 11^{*}$ & $78 \pm 11^{*}$ & $77 \pm 10$ & 0.014 \\
\hline $\mathrm{FBG}, \mathrm{mmol} / \mathrm{l}$ & $5.4 \pm 1.4$ & $5.7 \pm 1.7^{\star}$ & $5.7 \pm 1.7^{\star}$ & $6.0 \pm 1.7^{\star}$ & $<0.001$ \\
\hline $2 \mathrm{hBG}, \mathrm{mmol} / \mathrm{l}$ & $6.7 \pm 2.5$ & $7.6 \pm 2.9^{*}$ & $7.6 \pm 2.9^{*}$ & $8.9 \pm 3.7^{*}$ & $<0.001$ \\
\hline HOMA-IR & $1.2(0.8 \sim 1.9)$ & $1.8(1.2 \sim 2.5)^{\star}$ & $1.8(1.2 \sim 2.5)^{*}$ & $2.5(1.7 \sim 3.9)^{\star}$ & $<0.001$ \\
\hline $\mathrm{TG}, \mathrm{mmol} / \mathrm{l}$ & $1.2(0.9 \sim 1.8)$ & $1.4(1.0 \sim 2.0)^{*}$ & $1.4(1.0 \sim 2.0)^{\star}$ & $1.6(1.2 \sim 2.2)^{*}$ & $<0.001$ \\
\hline $\mathrm{TC}, \mathrm{mmol} / \mathrm{l}$ & $4.6 \pm 0.8$ & $4.7 \pm 0.8^{\star}$ & $4.7 \pm 0.8^{*}$ & $4.7 \pm 0.8^{\star}$ & 0.049 \\
\hline $\mathrm{HDL}-\mathrm{C}, \mathrm{mmol} / \mathrm{I}$ & $1.40 \pm 0.37$ & $1.26 \pm 0.29^{*}$ & $1.26 \pm 0.29^{\star}$ & $1.20 \pm 0.25^{\star}$ & $<0.001$ \\
\hline LDL-C,mmol/I & $2.59 \pm 0.72$ & $2.75 \pm 0.74$ & $2.75 \pm 0.74$ & $2.72 \pm 0.72^{*}$ & 0.004 \\
\hline LFC, \% & $3.9(1.9 \sim 7.8)$ & $5.0(2.3 \sim 10.8)^{*}$ & $5.0(2.3 \sim 10.8)^{\star}$ & $6.3(2.6 \sim 13.3)^{*}$ & $<0.001$ \\
\hline $\operatorname{NAFLD}(\mathrm{n}, \%)$ & $68,19.9 \%$ & $107,31.2 \%^{*}$ & $107,31.2 \%^{*}$ & $141,41.2 \%^{*}$ & $<0.001$ \\
\hline $\mathrm{DM}(\mathrm{n}, \%)$ & $42,12.3 \%$ & $81,23.6 \%^{*}$ & $81,23.6 \%{ }^{*}$ & $123,35.8 \%^{*}$ & $<0.001$ \\
\hline
\end{tabular}

The quartiles of ASM\% in were devided as follows, male: Q1: $\geq 32.0 \%$, Q2: $\geq 30.5 \% \sim<32.0 \%$, Q3: $\geq 29.0 \% \sim<30.5 \%$, Q4:<29.0\%; female: Q1: $\geq 26.8 \%$, Q2: $\geq 25.5 \% \sim<26.8 \%$, Q3: $\geq 24.3 \% \sim<25.5 \%$, Q4: $<24.3 \%$.

*: $\mathrm{P}<0.05$, compared with Q1 


\section{Quartiles of ASM\%}

Male

(n)

Female

Q1 n = 649

Q2 $n=651$

$60.8 \pm 8.7$

$61.7 \pm 8.8^{*}$

$55.1 \pm 8.1$

$58.0 \pm 8.3^{*}$

$23.5 \pm 2.9^{*}$

$22.5 \pm 4.2^{*}$

$36.3 \pm 2.9^{*}$

$25.7 \pm 0.8^{*}$

$132 \pm 19^{*}$

$74 \pm 10^{*}$

$5.4 \pm 1.2^{*}$

$7.2 \pm 2.5^{\star}$

$1.6(1.0 \sim 2.3)$

$1.8(1.2 \sim 2.6)^{*}$

$1.2(0.9 \sim 1.8)$

$1.4(1.0 \sim 2.0)^{*}$

$5.3 \pm 0.9$

$5.2 \pm 0.8$

$1.64 \pm 0.42$

$1.54 \pm 0.37^{*}$

$2.86 \pm 0.75$

$3.02 \pm 0.79^{*}$

$4.8(2.5 \sim 9.7)$

$5.8(2.7 \sim 11.6)^{*}$

$\operatorname{NAFLD}(\mathrm{n}, \%)$

171(26.3\%)

LDL-C,mmol/I

LFC, \%
$80.5 \pm 8.6^{\star}$

$214(32.9 \%)^{*}$

Q3 n = 343

Q4

$$
\mathrm{n}=\mathbf{3 4 2}
$$

Q3 n = 649

Q4

$n=650$

$62.9 \pm 9.4^{*}$

$64.8 \pm 9.7^{\star}$

$<0.001$

$59.8 \pm 8.6^{*}$

$62.6 \pm 9.7^{*}$

$<0.001$

$24.4 \pm 3.1^{*}$

$26.0 \pm 3.7^{\star}$

$<0.001$

$82.8 \pm 8.5^{*}$

$87.5 \pm 9.4^{\star}$

$<0.001$

$24.8 \pm 4.8^{*}$

$27.6 \pm 5.8^{*}$

$<0.001$

$38.8 \pm 3.0^{*}$

$42.4 \pm 3.4^{\star}$

$<0.001$

$24.5 \pm 0.6^{*}$

$22.8 \pm 1.1^{*}$

$<0.001$

$135 \pm 20^{*}$

$138 \pm 19^{*}$

$<0.001$

$75 \pm 9$

$76 \pm 10^{*}$

$<0.001$

$5.5 \pm 1.3^{*}$

$5.7 \pm 1.7^{*}$

$7.7 \pm 4.0^{*}$

$8.3 \pm 3.4^{*}$

$2.1(1.4 \sim 3.2)^{*}$

$2.3(1.6 \sim 3.6)^{\star}$

$<0.001$

$1.5(1.1 \sim 2.1)$

$1.6(1.2 \sim 2.2)^{*}$

$<0.001$

$5.3 \pm 0.9$

$5.4 \pm 1.0^{*}$

$<0.001$

$1.48 \pm 0.34$

$1.46 \pm 0.33^{*}$

$<0.001$
$3.04 \pm 0.83^{*}$

$5.9(2.7 \sim 11.8)^{*}$

$219(33.7 \%)^{*}$
$3.14 \pm 0.86^{*}$

$7.1(3.2 \sim 13.6)^{*}$

$275(42.3 \%)^{*}$
$<0.001$

$<0.001$

$<0.001$

The quartiles of ASM\% in were devided as follows, male: Q1: $\geq 32.0 \%, \mathrm{Q} 2: \geq 30.5 \% \sim<32.0 \%, \mathrm{Q} 3$ : $\geq 29.0 \% \sim<30.5 \%$, Q4:<29.0\%; female: Q1: $\geq 26.8 \%$, Q2: $\geq 25.5 \% \sim<26.8 \%$, Q3: $\geq 24.3 \% \sim<25.5 \%$, Q4: $<24.3 \%$.

*: $\mathrm{P}<0.05$, compared with Q1 


\begin{tabular}{|c|c|c|c|c|c|}
\hline \multicolumn{5}{|c|}{ Quartiles of ASM\% } & \multirow{3}{*}{$\begin{array}{l}P \\
\text { value }\end{array}$} \\
\hline \multirow[t]{2}{*}{ Male } & Q1 n = 343 & Q2 n = 343 & Q3 n = 343 & Q4 & \\
\hline & & & & $n=342$ & \\
\hline $\mathrm{DM}(\mathrm{n}, \%)$ & $88,13.6 \%$ & $106,16.3 \%$ & $152,23.4 \%{ }^{*}$ & $158,24.3 \%^{*}$ & $<0.001$ \\
\hline \multicolumn{6}{|c|}{$\begin{array}{l}\text { The quartiles of ASM\% in were devided as follows, male: Q1: } \geq 32.0 \% \text {, Q2: } \geq 30.5 \% \sim<32.0 \% \text {, Q3: } \\
\geq 29.0 \% \sim<30.5 \%, \text { Q4: }<29.0 \% \text {; female: Q1: } \geq 26.8 \%, \text { Q2: } \geq 25.5 \% \sim<26.8 \%, \text { Q3: } \geq 24.3 \% \sim<25.5 \%, \text { Q } \\
<24.3 \% \text {. }\end{array}$} \\
\hline \multicolumn{6}{|c|}{ *: P<0.05, compared with Q1 } \\
\hline
\end{tabular}

\section{Effects of skeletal muscle mass on glucose metabolism and other metabolic parameters}

The spearman analysis showed that besides age, body composition and metabolic parameters, including BMI, FM, FM\%, TG, LDL-C, SBP and DBP, ASM\% had a negative correlation with FBG, 2hBG, HOMA-IR and LFC (all $P<0.001)$. (Table 2). 
Table 2

Spearman analysis of ASM\% and other clinical parameters

\begin{tabular}{|c|c|c|}
\hline & ASM\% & \\
\hline & Male (r, P value) & Female ( $r, P$ value) \\
\hline Age(years) & $-0.317,<0.001$ & $-0.164,<0.001$ \\
\hline $\mathrm{BMI}\left(\mathrm{kg} / \mathrm{m}^{2}\right)$ & $-0.347,<0.001$ & $-0.441,<0.001$ \\
\hline WC (cm) & $-0.452,<0.001$ & $-0.448,<0.001$ \\
\hline $\mathrm{FM}(\mathrm{kg})$ & $-0.605,<0.001$ & $-0.590,<0.001$ \\
\hline FM (\%) & $-0.792,<0.001$ & $-0.799,<0.001$ \\
\hline $\mathrm{FBG}(\mathrm{mmol} / \mathrm{L})$ & $-0.177,<0.001$ & $-0.106,<0.001$ \\
\hline $2 \mathrm{~h} \mathrm{BG}(\mathrm{mmol} / \mathrm{L})$ & $-0.254,<0.001$ & $-0.201,<0.001$ \\
\hline HOMA-IR & $-0.385,<0.001$ & $-0.264,<0.001$ \\
\hline TG (mmol/L) & $-0.198,<0.001$ & $-0.193,<0.001$ \\
\hline $\mathrm{TC}(\mathrm{mmol} / \mathrm{L})$ & $-0.049,0.071$ & $-0.087,<0.001$ \\
\hline $\mathrm{HDL}-\mathrm{C}(\mathrm{mmol} / \mathrm{L})$ & $0.213,<0.001$ & $0.162,<0.001$ \\
\hline LDL-C (mmol/L) & $-0.075,0.006$ & $-0.119,<0.001$ \\
\hline SBP (mm Hg) & $-0.244,<0.001$ & $-0.192,<0.001$ \\
\hline DBP (mm Hg) & $-0.075,0.006$ & $-0.139,<0.001$ \\
\hline LFC (\%) & $-0.149,<0.001$ & $-0.113,<0.001$ \\
\hline
\end{tabular}

To further investigate whether low ASM\% was associated with the risk of DM, we performed logistic stepwise regression analysis, with ASM\% quartiles as independent variate and the presence of DM as dependent variate. As shown in Table 3, a crude analysis showed that the odds ratios (ORs) for DM were $0.665(0.592-0.746)$ in male and $0.775(0.710-0.84)$ in female. The relationship remained significant in male after successively adjusting for age, cigarette smoking, family history of diabetes, $\mathrm{FM}, \mathrm{FM} \times \mathrm{ASM} \%$, obesity, SBP, TG, HDL-C and HOMA-IR, with ORs 0.537 (0.312-0.923), but the association disappeared in female (ORs 0.985 [0.614-1.580]). 
Table 3

Multivariate-adjusted associations of ASM\% quartiles with DM

\begin{tabular}{|lll|}
\hline & Male & Female \\
\hline & OR $(95 \% \mathrm{Cl}$, P value $)$ & OR $(95 \% \mathrm{Cl}, \mathrm{P}$ value $)$ \\
\hline unadjusted & 0.665 & 0.775 \\
& $(0.592-0.746,<0.001)$ & $(0.710-0.847,<0.001)$ \\
\hline Model 1 & 0.527 & 0.505 \\
& $(0.336-0.826,0.005)$ & $(0.342-0.745,0.001)$ \\
\hline Model 2 & 0.640 & 0.728 \\
& $(0.401-1.020,0.051)$ & $(0.481-1.101,0.133)$ \\
\hline Model 3 & 0.537 & 0.985 \\
& $(0.312-0.923,0.024)$ & $(0.614-1.580,0.950)$ \\
\hline Model 1: adjusted for age, cigarette smoking, diabetes family history, FM, FM×AS & \\
\hline M\% quartiles, obesity. & \\
\hline Model 2: adjusted for covariates in Model 1 plus SBP, TG, HDL-C.
\end{tabular}

\section{Effect of NAFLD on the relationship between skeletal muscle mass and DM}

As we know, NAFLD increased the prevalence and risk of type 2 diabetes, we also found ASM\% was negative associated with liver fat content in our study. Thus, we further conducted logistic analysis to observe the effect of NAFLD on the relationship between skeletal muscle mass and the risk of DM. As shown in Table 4. Among the 2658 non-NAFLD participants, the ASM\% quartile was negatively correlated with the risk of DM both in male and female before adjustment. After multiple adjustments, the negative association remained significant in male (ORs 0.330 [0.157-0.694]), but not in female (ORs 0.800 [0.416-1.537]). However, in the 1311 NAFLD patients, the results showed the correlation between ASM\% and DM dissociated after adjustments in both genders. 
Table 4

Multivariate-adjusted associations of ASM\% quartiles with DM in participants with or without NAFLD

Male Female

OR (95\%Cl, P value)

OR ( $95 \% \mathrm{Cl}, \mathrm{P}$ value)

Non-NAFLD $(n=2658)$

unadjusted

0.635

0.770

$(0.548-0.736,<0.001)$

$(0.682-0.870,<0.001)$

Model 1

0.403

0.581

$(0.221-0.735,0.003)$

(0.344-0.981,0.042)

Model 2

0.455

0.807

$(0.246-0.842,0.012)$

$(0.463-1.409,0.452)$

Model 3

0.330

0.800

$(0.157-0.694,0.003)$

$(0.416-1.537,0.503)$

\section{$\operatorname{NAFLD}(n=1311)$}

unadjusted

0.789

0.845

$(0.650-0.956,0.016)$

$(0.739-0.966,0.014)$

Model 1

1.259

0.508

$(0.542-2.924,0.592)$

(0.265-0.975,0.042)

Model 2

1.954

0.710

$(0.788-4.851,0.148)$

$(0.355-1.418,0.332)$

Model 3

1.328

1.106

$(0.435-4.055,0.619)$

$(0.485-2.523,0.810)$

Model 1: adjusted for age, cigarette smoking, diabetes family history, FM, FM×ASM\% quartiles, obesity.

Model 2: adjusted for covariates in Model 1 plus SBP, TG, HDL-C.

Model 3: adjusted for covariates in Model 2 plus HOMA-IR.

As NAFLD was diagnosed by LFC in the present study, which was displayed as continuous variable. We further stratified the population by LFC quartiles from low to high. The results showed that before adjustments, in the first three LFC quartiles in male and the first two LFC quartiles in female, the ASM\% quartiles were negatively correlated with the risk of DM. After adjustments, the relationship remained 
significant in the first and second quartile in male, however, the correlation no longer existed after adjustments in the third and forth quartile in male and in all quartiles in female. (Table 5)

Table 5

Multivariate-adjusted associations of ASM\% quartiles with DM in participants with different LFC

\begin{tabular}{|c|c|c|c|}
\hline LFC & DM & $\begin{array}{l}\text { OR } \\
\text { (95\% Cl, } \mathrm{P} \text { value) }\end{array}$ & $\begin{array}{l}\text { OR } \\
\text { (95\%Cl, P value) }\end{array}$ \\
\hline Male & $\mathrm{n}(\%)$ & Unadjusted & After adjusted \\
\hline \multirow[t]{2}{*}{ Q1 (n = 389) } & $82(21.1 \%)$ & 0.635 & 0.308 \\
\hline & & $(0.504-0.799,<0.001)$ & $(0.102-0.932,0.032)$ \\
\hline \multirow[t]{2}{*}{ Q2 (n = 350) } & $66(18.8 \%)$ & 0.540 & 0.184 \\
\hline & & $(0.416-0.700,<0.001)$ & $(0.049-0.685,0.012)$ \\
\hline \multirow[t]{2}{*}{ Q3 (n = 306) } & $74(24.2 \%)$ & 0.760 & 0.678 \\
\hline & & $(0.599-0.963,0.023)$ & $(0.158-2.908,0.601)$ \\
\hline Q4 & $116(35.7 \%)$ & 0.830 & 1.561 \\
\hline$(n=325)$ & & $(0.665-1.035,0.099)$ & $(0.440-5.539,0.491)$ \\
\hline \multicolumn{4}{|l|}{ Female } \\
\hline \multirow[t]{2}{*}{ Q1 $(n=605)$} & $84(13.9 \%)$ & 0.828 & 0.964 \\
\hline & & $(0.672-1.021,0.077)$ & $(0.311-2.990,0.949)$ \\
\hline \multirow[t]{2}{*}{ Q2 (n = 642) } & $92(14.3 \%)$ & 0.745 & 0.517 \\
\hline & & $(0.609-0.913,0.004)$ & $(0.170-1.578,0.247)$ \\
\hline \multirow[t]{2}{*}{ Q3 (n = 685) } & $118(17.2 \%)$ & 0.739 & 0.750 \\
\hline & & $(0.615-1.887,0.091)$ & $(0.274-2.048,0.574)$ \\
\hline \multirow[t]{2}{*}{ Q4 (n= 667) } & $210(31.5 \%)$ & 0.871 & 1.122 \\
\hline & & $(0.750-1.011,0.070)$ & $(0.445-2.830,0.808)$ \\
\hline \multicolumn{4}{|c|}{ 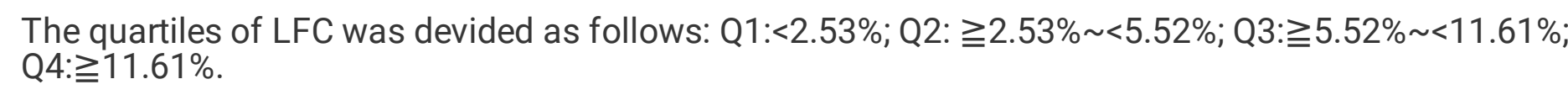 } \\
\hline
\end{tabular}

\section{Discussion}


As far as we know, several studies explored the relationship between skeletal muscle mass with diabetes. The cross-section research by Srikanthan et al. revealed skeletal muscle mass accessed by BIA was negatively associated with HOMA-IR and glycated hemoglobin A1c (HbA1C) in American middle aged population ${ }^{[23]}$. The cohort study conducted by Korean researchers also showed lower ASM\% could increased the risk of DM in young and middle-aged population ${ }^{[24,25]}$. Although these studies showed results similar to our findings, they didn't showed the gender difference of the relationship between muscle loss and DM. In our study, we found that skeletal muscle mass measured by ASM\% was negatively associated with blood glucose, and skeletal muscle loss may increase the risk of DM only in men. The dissociation of skeletal muscle mass loss and DM in women is noteworthy, especially after adjusted by fat mass and lipid parameters. The exact reason for this interesting phenomenon is still unknown. The subjects in the present study were older., and previous studies showed with age increasing, the percentage of body fat increased gradually, which was more pronounced in older ${ }^{[24,25]}$ and women ${ }^{[26]}$. This could result increasing insulin resistance and lipid disorders more significant in women, which might neutralize the effect of skeletal muscle reduction on diabetes. Another possible explanation is the difference of body fat distribution between genders. Although the body fat percentage of women was higher than the match aged men, and with aging the accumulation of intramuscular and intermuscular fat was more significant in women than in men ${ }^{[27]}$, however, women had more type I muscle fibers than men, contributing stronger oxidative function of skeletal muscle ${ }^{[28]}$, which could reduce the risk of diabetes. On the other hand, hormones especially estrogen could influence the metabolism of triglyceride and free fatty acids ${ }^{[29]}$. Estrogen decreased as aging, especially in postmenopausal women, which may result in the reduction of triglyceride, and was associated with the reduced risk of diabetes ${ }^{[30]}$. In addition, in the process of aging, the decline of skeletal muscle mass was more remarkable in male than in female, which also contributed to the more significant effect of skeletal muscle loss on the risk of diabetes in male. The results suggest gender stratified management of diabetes according to skeletal muscle needs to be considered in the future. Increasing skeletal muscle mass might have a more beneficial effect on improving glucose metabolism in male population.

NAFLD is an important risk factor of diabetes, and several previous studies have demonstrated that low skeletal muscle mass was also independently associated with NAFLD ${ }^{[14-16]}$. In the present study, the skeletal muscle mass was also negatively associated with LFC, which was similar to the previous results. Whether liver fat content would influence the relationship between sarcopenia and diabetes is still unknown. Our results showed the relationship between skeletal muscle mass and diabetes existed in the non-NAFLD male population and disappeared in the NAFLD population. It indicated that as an important risk factor for diabetes, excessive liver fat accumulation which could lead to insulin resistance, mitochondrial dysfunction, hyperlipidemia and so on ${ }^{[31]}$, could lessen the effect of skeletal muscle reduction on diabetes. The results indicated that reducing liver fat maybe more important when referred to improving diabetes in NAFLD population. While in the non-NAFLD population, skeletal muscle mass enhancement might be helpful in diabetes treatment. Further analysis in our study revealed an interesting result was that, the association of skeletal muscle and DM remained in the male population whose LFC 
less than $5.52 \%$, which was similar to the histopathologic diagnosis of fatty liver, The findings indicated that in men with liver fat less than $5.52 \%$, increasing skeletal muscle mass may help prevent diabetes.

The mechanism underlying the relationship between low skeletal muscle mass and diabetes are still not fully understood. It has been known that insulin resistance and systemic inflammation played an important role on the development of both skeletal muscle reduction and diabetes ${ }^{[6,32]}$. As an important target organ of insulin action, skeletal muscle plays an important role in maintaining the basic metabolism and glucose metabolism stability ${ }^{[33]}$. Decreased skeletal muscle mass, which was often accompanied by intermuscular fat accumulation, could increase macrophage infiltration, mitochondrial dysfunction and inflammatory factors releasing, contributing to insulin resistance and reduced glucose uptake and utilization ${ }^{[34-36]}$. While increasing skeletal muscle mass could improve insulin sensitivity and glucose metabolism ${ }^{[23]}$. But our current study also found that in male population, age-related skeletal muscle mass loss was independently associated with the risk of diabetes after adjustment for obesity, HOMA-IR and all components of metabolic syndrome, which suggested that there may be other mechanisms to explain this association. Although it is unclear whether skeletal muscle loss is the cause or the consequence of diabetes, a direct crosstalk between skeletal muscle and glucose metabolism has been uncovered. Previous studies have shown that skeletal muscle can secrete a variety of cytokines, such as IL-6 and Irisin, which can regulate insulin sensitivity, promote glucose uptake by skeletal muscle cells, reduce liver gluconeogenesis, and improve glucose metabolism by acting on adipose tissue, liver

and other tissues ${ }^{[37,38]}$. The impairment of muscle secretary function due to muscle loss may contribute to the development of diabetes.

To our knowledge, the current study might be the first large-scale community population research to access the influence of NAFLD on the association between diabetes with gender- and age-related skeletal muscle mass which was measured by DXA, a more accurated method of body compostion recommended by the guidelines ${ }^{[39]}$.Our findings might develop a new perspective for preventing diabetes, especially in male non-NAFLD population. But there are also several limitations in our study. First, it was a crosssectional study which cannot reveal the causal relationship between skeletal muscle mass and diabetes. So it is necessary to further verify our findings in a prospective cohort study. The second, the association of skeletal muscle loss and diabetes only existed in the first and second LFC quartiles, and the cut-off point of LFC should be further conformed. Second, several serum myokines were not detected in our current study, which might help explore the mechanisms underlying the relationship between low skeletal muscle mass and diabetes.

\section{Conclusions}

Skeletal muscle loss was associated with increasing risk of diabetes in male non-NAFLD middle-aged and elderly community population. Our results suggested a new practical strategy to facilitate personalised intervention of diabetes by increasing skeletal muscle mass in male non-NAFLD patients. 


\section{Abbreviations}

NAFLD non alcoholic fatty liver disease

NASH non-alcoholic steatohepatitis

DM diabetes mellitus

LFC liver fat content

DXA dual energy X-ray absorption

FBG fasting blood-glucose

2hBG 2-hour postprandial blood glucose

HOMA-IR homeostasis model assessment for insulin resistance

BMI body mass indes

TC total cholesterol

TG triglyceride

LDL-C low density lipoprotein cholesterol

HDL-C high density lipoprotein cholesterol

OGTT oral glucose tolerance test

ASM appendicular skeletal mass

SBP systolic pressure

DBP diastolic pressure

HbA1C glycated hemoglobin A1c

\section{Declarations}

\section{Acknowledgements}

We are grateful to Ting Liu and Jing Li for their kind help in the sample and data collection for our study.

\section{Funding}


This work was supported by the National Key Basic Research Program of China (Grant No.

2012CB524906 to Gao X and Grant No. 2011CB504004 to Gao X), the Shanghai Municipal Health Bureau Foundation (Grant No.12GWZX0103 to Gao X), the Science and Technology Commission of Shanghai Municipality (10411956400 to Gao X), the National Ministry of Education Program (985 III-YFX0302 to Gao X) .The funding sources play an important role on the design of the study and collection, analysis, and interpretation of data and writing the manuscript.

\section{Author Contributions}

Conceived and designed the experiments: Xin Gao; Performed the experiments: Huandong Lin, Lingyan Chen, Li Wu, Qian Li, Hui Ma, Yu Hu; Collected and analyzed the data: Lingyan Chen, Mingfeng Xia; Wrote the paper: Lingyan Chen; Performed laboratory examination: Lingyan Chen, Li Wu, Qian Li. All authors approved the final version. Huandong Lin and Xin Gao are the guarantor of this work and has full access to all of the data, take responsibility for the integrity of the data, and control the decision to publish.

\section{Ethics approval and consent to participate}

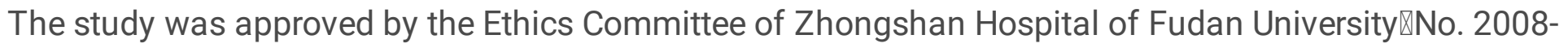
119囚. All the subjects have given their written informed consent.

\section{Consent for publication}

Both the authors and the participants agreed with the publication.

\section{Availability of data and materials}

The datasets generated during and/or analysed during the current study are available from the corresponding author on reasonable request.

\section{Competing interests}

The authors declared that they have no conflict interests.

\section{References}

1 Yamada M, Moriguch Y, Mitani T, Aoyama T, Arai H. Age-dependent changes in skeletal muscle mass and visceral fat area in Japanese adults from 40 to 79 years-of-age [J].Geriatr Gerontol Int 2014, 14 (Suppl. 1): 8-14. DOI: 10.1111/ggi.12209.

2 Psutka SP, Carrasco A, Schmit GD, Moynagh MR, Boorjian SA, Frank I, et al. Sarcopenia in patients with bladder cancer undergoing radical cystectomy: impact on cancer-specific and all-cause mortality[J]. Cancer. 2014, 120(18):2910-8. DOI: 10.1002/cncr.28798. 
3 Biolo G, Cederholm T, Muscaritoli M. Muscle contractile and metabolic dysfunction is a common feature of sarcopenia of aging and chronic diseases: from sarcopenic obesity to cachexia[J]. Clin Nutr. 2014, 33(5):737-48. DOI: 10.1016/j.clnu.2014.03.007.

4 Bhanji RA, Carey EJ, Yang L, Watt KD. The Long Winding Road to Transplant: How Sarcopenia and Debility Impact Morbidity and Mortality on the Waitlist[J]. Clin Gastroenterol Hepatol 2017, 15(10): 14921497. DOI: 10.1016/j.cgh.2017.04.004.

5 Meyer MM , Levin K, Grimmsmann T, Beck-Nielsen H, Klein HH. Insulin Signalling in Skeletal Muscle of Subjects With or Without Type II-diabetes and First Degree Relatives of Patients With the Disease[J]. Diabetologia, 2002, 45 (6): 813-22. DOI: 10.1007/s00125-002-0830-9.

6 Kim TN, Park MS, Lim KI, Choi HY, Yang SJ, Yoo HJ, et al. Relationships between sarcopenic obesity and insulin resistance, inflammation, and vitamin D status: the Korean Sarcopenic Obesity Study[J]. Clin Endocrinol (Oxf), 2013, 78(4), 525-532. DOI: 10.1111/j.1365-2265.2012.04433.x.

7 Kim KS, Park KS, Kim MJ, Kim SK, Cho YW, Park SW. Type 2 Diabetes Is Associated With Low Muscle Mass in Older Adults[J]. Geriatr Gerontol Int,2014,14 Suppl 1:115-21. DOI: 10.1111/ggi.12189.

8 Kim TN, Park MS, Yang SJ, Yoo HJ, Kang HJ, Song W, et al. Prevalence and determinant factors of sarcopenia in patients with type 2 diabetes[J]. Diabetes Care. 2010;33(7):1497. DOI: 10.2337/dc09-2310.

9 Son JW, Lee SS, Kim SR, Yoo SJ, Cha BY, Son HY, et al. Low muscle mass and risk of type 2 diabetes in middle-aged and older adults: findings from the KoGES[J]. Diabetologia. 2017;60(5):865-872. DOI: 10.1007/s00125-016-4196-9.

10 Larsen BA, Wassel CL, Kritchevsky SB, Strotmeyer ES, Criqui MH, Kanaya AM, et al. Association of Muscle Mass, Area, and Strength With Incident Diabetes in Older Adults: The Health ABC Study[J]. J Clin Endocrinol Metab. 2016, 101(4): 1847-1855. DOI: 10.1210/jc.2015-3643.

11 Dai W, Ye Y, Liu AZ, Wen SW, Deng J, Wu X, et al. Prevalence of Nonalcoholic Fatty Liver Disease in Patients With Type 2 Diabetes Mellitus: A Meta-Analysis[J]. Medicine (Baltimore),2017, 96 (39), e8179. DOI: 10.1097/MD.0000000000008179.

12 National Workshop on Fatty Liver and Alcoholic Liver Disease, Chinese Society of Hepatology, Chinese Medical Association Fatty Liver Expert Committee, Chinese Medical Doctor Association. Guideline of prevention and treatment for nonalcoholic fatty liver disease: a 2018 update [J]. J Mod Med Health, 2018, 34(5) :641-649.

13 Park SK, Seo MH, Shin HC, Ryoo JH. The clinical availability of non-alcoholic fatty liver disease as an early predictor of type 2 diabetes mellitus in korean men: 5-years' prospective cohort study [J]. Hepatology. 2012; 57: 1378-1383. DOI: 10.1002/hep.26183. 
14 Hong HC, Hwang SY, Choi HY, Yoo HJ, Seo JA, Kim SG, et al. Relationship between Sarcopenia and nonalcoholic fatty liver disease: the Korean Sarcopenic Obesity Study. Hepatology. 2014, 59(5):17721778. DOI: 10.1002/hep.26716.

15 Koo BK, Kim D, Joo SK, Kim JH, Chang MS, Kim BG, et al. Sarcopenia is an independent risk factor for non-alcoholic steatohepatitis and significant fibrosis [J]. Hepatology, 2017, 66:123-31. DOI:

10.1016/j.jhep.2016.08.019.

16 Kim G, Lee SE, Lee YB, Jun JE, Ahn J, Bae JC, et al. Relationship Between Relative Skeletal Muscle Mass and Nonalcoholic Fatty Liver Disease: A 7-Year Longitudinal Study[J]. Hepatology, 2018, 68(5): 1755-1768. DOI: 10.1002/hep.30049.

17 Gao X, Hofman A, Hu YU, Lin HD, Zhu CW, Jeekel J, et al. The Shanghai Changfeng Study: a community-based prospective cohort study of chronic diseases among middle-aged and elderly: objectives and design[J]. Eur J Epidemiol. 2010, 25:885-893. DOI: 10.1007/s10654-010-9525-6.

18 Lin HD, Li Q, Hu Y, Zhu CW, Ma H, Gao J, et al. The prevalence of multiple noncommunicable diseases among middle-aged and elderly people: the Shanghai Changfeng Study[J]. Eur J Epidemiol. 2017,32(2):159-163. DOI: 10.1007/s10654-016-0219-6.

19 Xia MF, Yan HM, He WY, Li XM, Li CL, Yao XZ, et al. Standardized ultrasound hepatic/renal ratio and hepatic attenuation rate to quantify liver fat content:an improvement method[J]. Obesity. 2012, 20: 444452. DOI: 10.1038/oby.2011.302.

20 Kim YS, Lee Y, Chung YS, Lee DJ, Joo NS, Hong D, et al. Prevalence of sarcopenia and sarcopenic obesity in the Korean population based on the Fourth Korean National Health and Nutritional Examination Surveys[J]. J Gerontol A Biol Sci Med Sci 2012, 67: 1107-1113. DOI:

10.1093/gerona/gls071.

21 Chen CM, Kong LZ. Chinese Guidelines for the prevention and Control of overweight and obesity in adults [M]. Beijing: People's Medical Publishing House, 2006.

22 ORGANIZATION WHO. Definition, diagnosis and classification of diabetes mellitus and its complications: report of a WHO consultation. Part 1: diagnosis and classification of diabetes mellitus. [M]. Department of Noncommunicable Disease Surveillance Geneva. Geneva: WHO 1999.

23 Srikanthan P, Karlamangla AS. Relative muscle mass is inversely associated with insulin resistance and prediabetes. Findings from the third National Health and Nutrition Examination Survey[J]. J Clin Endocrinol Metab. 2011; 96:2898-2903. DOI: 10.1210/jc.2011-0435.

24 Hong S, Chang Y, Jung HS, Yun KE, Shin H, Ryu S.. Relative muscle mass and the risk of incident type 2 diabetes: A cohort study[J]. PLoS One. 2017, 12(11): e0188650. DOI: 10.1371/journal.pone.0188650. 
25 Son JW, Lee SS, Kim SR, Yoo SJ, Cha BY, Son HY, et al. Low muscle mass and risk of type 2 diabetes in middle-aged and older adults: findings from the KoGES. Diabetologia, 2017, 60:865-872. DOI:

10.1007/s00125-016-4196-9.

26 Lu T, Wang Y, Ting Dou T, Xue B, Tan Y, Yang J.. Pancreatic Fat Content Is Associated With $\beta$-Cell Function and Insulin Resistance in Chinese Type 2 Diabetes Subjects[J].Endocr J, 2019,66 (3), 265-270. DOI: 10.1507/endocrj.EJ18-0436.

27 Beaudry KM, Devries MC. Sex-based Differences in Hepatic and Skeletal Muscle Triglyceride Storage and Metabolism 1[J]. Appl Physiol Nutr Metab, 2019, 44 (8): 805-813. DOI: 10.1139/apnm-2018-0635.

28 Lundsgaard AM, Kiens B. Gender Differences in Skeletal Muscle Substrate Metabolism-Molecular Mechanisms and Insulin Sensitivity[J]. Front Endocrinol (Lausanne), 2014, 5:195. DOI: 10.3389/fendo.2014.00195.

29 Palmisano BT, Zhu L, Eckel RH, Stafford JM. Sex differences in lipid and lipoprotein metabolism[J]. Mol Metab. 2018;15:45-55. DOI: 10.1016/j.molmet.2018.05.008.

30 Muka T, Nano J, Jaspers L, Meun C, Bramer WM, Hofman A,et al. Associations of Steroid Sex Hormones and Sex Hormone-Binding Globulin With the Risk of Type 2 Diabetes in Women: A PopulationBased Cohort Study and Meta-analysis[J]. Diabetes 2017, 66(3):577-586. DOI: 10.2337/db16-0473.

31 Tilg H, Moschen AR, Roden M. NAFLD and Diabetes Mellitus [J]. Nat Rev Gastroenterol Hepatol, 2017, 14 (1), 32-42. DOI: 10.1038/nrgastro.2016.147.

32 Mesinovic J, Zengin A, Courten BD, Ebeling PR, Scott D. Sarcopenia and type 2 diabetes mellitus:a bidirectional relationship [J]. Diabetes, Metabolic Syndrome and Obesity: Targets and Therapy 2019,12:1057-1072. DOI: 10.2147/DMSO.S186600.

33 Thiebaud D, Jacot E, DeFronzo RA, Maeder E, Jequier E, Felber JP. The effect of graded doses of insulin on total glucose uptake, glucose oxidation, and glucose storage in man[J]. Diabetes, 1982, 31(11) :957-963. DOI: 10.2337/diacare.31.11.957.

34 Ferrannini E, lozzo P, Virtanen KA, Honka MJ, Bucci M, Nuutila P. Adipose Tissue and Skeletal Muscle Insulin-Mediated Glucose Uptake in Insulin Resistance: Role of Blood Flow and Diabetes[J]. Am J Clin Nutr, 2018, 108 (4), 749-758. DOI: 10.1093/ajcn/nqy162.

35 Cleasby ME, Jamieson PM, Atherton PJ. Insulin resistance and sarcopenia: mechanistic links between common co-morbidities[J]. J Endocrinol, 2016, 229 (2), R67-81. DOI: 10.1530/JOE-15-0533.

36 Li XH, Li ZM, Zhao MH, Nie YX, Liu PS, Zhu YL, et al. Skeletal Muscle Lipid Droplets and the Athlete's Paradox [J]. Cells 2019, 8 (3). DOI: 10.3390/cells8030249. 
37 Pedersen BK, Febbraio MA. Muscles, Exercise and Obesity: Skeletal Muscle as a Secretory Organ[J]. Nat Rev Endocrinol, 2012, 8 (8):457-65. DOI: 10.1038/nrendo.2012.49.

38 Perakakis N, Triantafyllou GA, Fernández-Real JM, Huh JY, Park KH, Seufert J, et al. Physiology and Role of Irisin in Glucose Homeostasis [J]. Nat Rev Endocrinol, 2017,13(6):324-337. DOI:

10.1038/nrendo.2016.221.

39 Cruz-Jentoft AJ, Baeyens JP, Bauer JM, Boirie Y, Cederholm T, Landi F, et al. Sarcopenia: European consensus on definition and diagnosis[J]. Age and Ageing, 2010,39:412-423.DOI:

10.1093/ageing/afq034. 